\title{
MS44-P04 | EFFECTS OF X-RAY IRRADIATION AND THERMAL ANNEALING ON THE CO DOPANT LOCATION IN CO-DOPED TIO 2 NANOCRYSTALS
}

Soo, Yun-Liang (National Tsing Hua University, Hsinchu, TWN); Wu, Tai-Sing (National Tsing Hua University, Hsinchu, TWN); Jeng, Horng-Tay (National Tsing Hua University, Hsinchu, TWN); Chang, Shih-Lin (National Tsing Hua University, Hsinchu, TWN)

Extended $x$-ray absorption fine structure (EXAFS) and x-ray absorption near-edge structure (XANES) techniques have been employed to investigate the variations of dopant local structures due to $x$-ray irradiation and thermal annealing in Co-doped $\mathrm{TiO}_{2}$ nanocrystals. The Co dopant atoms were found to occupy $\mathrm{Ti}$ sites in the as-made nanocrystal sample. After thermal annealing at elevated temperatures, Co atoms were relocated to an interstitial site and the oxygen coordination around Co atoms changed from an octahedral coordination to a square-planar coordination. The UV-vis diffuse reflectance spectra also indicate a substantial band-gap change from $3.1 \mathrm{eV}$ to $2.3 \mathrm{eV}$ as a result of the local structural change. The variation of Co dopant location due to thermal annealing was then found to be reversed by x-ray irradiation. First-principle density functional theory (DFT) calculations were carried out to determine the formation energies and to verify the structural stability of the supercells associated with the two distinct Co local structures. Control of dopant locations and band-gap width using x-ray irradiation and thermal annealing may provide unprecedented opportunities for technological applications of the Co-doped $\mathrm{TiO}_{2}$ nanocrystal system. 